\title{
Small-World Network Effect in Competing Glauber- and Kawasaki-type Dynamics
}

\author{
Jian-Yang Zhu ${ }^{1,2, *}$,Wei $\mathrm{Liu}^{2}$, Han $\mathrm{Zhu}^{3}$ \\ ${ }^{1}$ CCAST (World Laboratory), Box 8730, Beijing 100080, China. \\ ${ }^{2}$ Department of Physics, Beijing Normal University, Beijing 100875, China. ${ }^{\dagger}$ \\ ${ }^{2}$ Department of Physics, Nanjing University, Nanjing 210093, China.
}

\begin{abstract}
In this article, we investigate the competing Glauber-type and Kawasaki-type dynamics with small-world network (SWN) effect, in the framework of the Gaussian model. The Glauber-type single-spin transition mechanism with probability $p$ simulates the contact of the system with a heat bath and the Kawasaki-type dynamics with probability $1-p$ simulates an external energy flux. Two different types of SWN effect are studied, one with the total number of links increased and the other with it conserved. The competition of the dynamics leads to an interesting self-organization process that can be characterized by a phase diagram with two identifiable temperatures. By studying the modification of the phase diagrams, the SWN effect on the two dynamics is analyzed. For the Glauber-type dynamics, more important is the altered average coordination number while the Kawasaki-type dynamics is enhanced by the long range spin interaction and redistribution.

Keywords: Small-World Network Effect; Classical Spin Model; Competing Glauber- and Kawasaki-type Dynamics; Phase Diagram
\end{abstract}

PACS number(s): 89.75.-k, 64.60.Ht, 64.60.Cn, 64.60.Fr

\section{INTRODUCTION}

Many systems in nature or society can be well described by small world networks (SWN), which were first proposed by Watts and Strogatz in Ref. [1]. SWNs are those intermediate between a regular lattice and a random network. They can be realized by introducing a very small portion of long range links to a regular lattice. Such networks appear as small-worlds like random graphs, i.e., with a small average node-node distance that grows logarithmically with the network size. Meanwhile they also have usually large clustering coefficients [2-4]. Since the first model system was proposed [1], a large literature has appeared and the properties of various models and processes on SWNs have been extensively studied, including percolation, coloring, coupled oscillators, iterated games, diffusion processes, epidemic processes, and spin models (see Ref. [2-4] and references therein). For example, it has been recently found that some spin-lattice models belonging to different universality classes now show mean-field behavior on SWNs [4-9]. Furthermore, recently Zhu et al. introduced SWN effect to critical dynamics [10], and the investigation has been extended to the kinetic properties of the spin models.

According to Glauber's and Kawasaki's theory [11,12], the time evolution of the order parameters can be described by a Markov process with a specific choice of the dynamic mechanism. Two mechanisms have been extensively studied: Glauber's single-spin flip mechanism [11] with the order parameter nonconserved and Kawasaki's spin-pair exchange mechanism [12] with the order parameter conserved. In the past decade, an interesting problem has been attracting much attention, i.e., the competing Glauber-type and Kawasaki-type dynamics. We briefly explain the physical ground: Glauber's mechanism with probability $p$ is used to simulate the contact of the system with a heat bath and favors a low energy state. Meanwhile Kawasaki's mechanism with probability $1-p$ simulates an external energy flux and, naturally, favors a high energy state. Then there will a competition between the two dynamics. This competing mechanism has been applied to the spin models [13-19], and interesting self-organization has been reported. All these works have considered regular lattice, and to extend the investigation to the small-world networks should be of interest. At the same time, such a study may also have theoretical meaning. In Zhu's work [10], it has been found that the SWN effect may have different types of influence on the Glauber-type dynamics and the Kawasaki-type dynamics. By focusing on the competition of the dynamics, it is easy for us to further understand the influence of the SWN effect and highlight the disparities between the dynamics.

In this article we investigate the SWN effect on the competing dynamics, and we demonstrate the conclusions by comparing the phase diagrams obtained with and without the SWN effect. Before we present the calculations, we

*Email address: zhujy@bnu.edu.cn

${ }^{\dagger}$ Mailing address 
first describe the prototypes of SWNs that are used in the present study, and then briefly review the generalized competing mechanism.

The SWN models: After the first prototype of SWN was proposed by Watts and Strogatz [1], there appear a variety of model systems that can be categorized into the two groups, i.e., with the total number of links increased or conserved. Correspondingly, in the present research we study two specific, while representative models described below: (1) in a one-dimensional loop, for example, each randomly selected pair of vertices are additionally connected with probability $p_{A} ;(2)$ the vertices are visited one after another, and each of its links in the clockwise sense is left in place with probability $1-p_{R}$ and is reconnected to a randomly selected other node with probability $p_{R}$. Networks of higher dimensions can be similarly built. We call the first model adding-type small-world network (A-SWN) and the second one rewiring-type network (R-SWN).

The generalized competing mechanism: Glauber's flip mechanism and Kawasaki's exchange mechanism were originally presented for the Ising model. Recently they have been generalized to single-spin transition mechanism [20] and spin-pair redistribution mechanism [21], respectively, which can be applied to arbitrary spin systems. Correspondingly there is the generalized competing dynamics [19], which provides a basis for the discussions in this article. With the competing mechanism, the master equation can be written as

$$
\frac{d}{d t} P(\{\sigma\}, t)=p G_{m e}+(1-p) K_{m e},
$$

where $p G_{m e}$ denotes the single-spin transition with probability $p$ and $(1-p) K_{m e}$ denotes the spin-pair redistribution with probability $1-p$. For your convenience we list in Sec. II and III some important equations of the transition mechanism and the redistribution mechanism, the details of which can be found in respective references.

In this article we study the generalized competing dynamics in the framework of the kinetic Gaussian model, which is mathematically tractable. It has been revealed in Ref. [10] that the SWN effect on the Gaussian model is of the mean-field nature. Considering this, in the present study a simplified method is adopted, although a rigorous treatment is possible.

In Sec. II we study the competing mechanics on A-SWN and In Sec. III, we extend the discussions to R-SWN. Section IV is the summarization with some discussions.

\section{COMPETING MECHANISM ON A-SWN}

In this section we present our discussion of the competing dynamics on adding-type small-world networks (the definition see the Introduction). In subsection II A through II C we give the formulation of the Glauber-type mechanism, the Kawasaki-type mechanism and the competing mechanism, and then in subsection IID the competing mechanism is applied to the three-dimensional Gaussian model.

\section{A. The Glauber-type mechanism}

With the Glauber-type single-spin transition mechanism $[10,20]$ on A-SWN, the master equation can be written as

$$
\frac{d}{d t} P(\{\sigma\}, t)=-\sum_{i} \sum_{\sigma}\left[W_{i}\left(\sigma_{i} \rightarrow \hat{\sigma}_{i}\right) P(\{\sigma\}, t)-W_{i}\left(\hat{\sigma}_{i} \rightarrow \sigma_{i}\right) P\left(\left\{\sigma_{j \neq i}\right\}, \hat{\sigma}, t\right)\right],
$$

where the transition probability

$$
W_{i}\left(\sigma_{i} \rightarrow \hat{\sigma}_{i}\right)=\frac{1}{Q_{i}} \exp \left[-\beta \mathcal{H}\left(\left\{\sigma_{j \neq i}\right\}, \hat{\sigma}_{i}\right)\right], Q_{i}=\sum_{\hat{\sigma}_{i}} \exp \left[-\beta \mathcal{H}\left(\left\{\sigma_{j \neq i}\right\}, \hat{\sigma}_{i}\right)\right] .
$$

With equation (2), one can continue to write that $[10,20]$,

$$
\frac{d}{d t} q_{k}(t)=Q_{k}^{G}=-q_{k}(t)+\sum_{\{\sigma\}}\left[\sum_{\hat{\sigma}_{k}} \hat{\sigma}_{k} W_{k}\left(\sigma_{k} \rightarrow \hat{\sigma}_{k}\right)\right] P(\{\sigma\}, t),
$$

where

$$
q_{k}(t)=\sum_{\{\sigma\}} \sigma_{k} P(\{\sigma\}, t)
$$




\section{B. The Kawasaki-type mechanism}

With the Kawasaki-type spin-pair redistribution mechanism on A-SWN, the master equation can be written as $[10,21]$

$$
\begin{aligned}
\frac{d}{d t} P(\{\sigma\}, t)= & \sum_{\langle j l\rangle} \sum_{\hat{\sigma}_{j} \hat{\sigma}_{l}}\left[-W_{j l}\left(\sigma_{j} \sigma_{l} \rightarrow \hat{\sigma}_{j} \hat{\sigma}_{l}\right) P(\{\sigma\}, t)+W_{j l}\left(\hat{\sigma}_{j} \hat{\sigma}_{l} \rightarrow \sigma_{j} \sigma_{l}\right) P\left(\left\{\sigma_{j \neq i}, \sigma_{l \neq k}\right\}, \hat{\sigma}_{j}, \hat{\sigma}_{l}, t\right)\right] \\
& +\frac{1}{2} p_{A} \sum_{j} \sum_{l \neq j} \sum_{\hat{\sigma}_{j} \hat{\sigma}_{l}}\left[-W_{j l}\left(\sigma_{j} \sigma_{l} \rightarrow \hat{\sigma}_{j} \hat{\sigma}_{l}\right) P(\{\sigma\}, t)\right. \\
& \left.+W_{j l}\left(\hat{\sigma}_{j} \hat{\sigma}_{l} \rightarrow \sigma_{j} \sigma_{l}\right) P\left(\left\{\sigma_{j \neq i}, \sigma_{l \neq k}\right\}, \hat{\sigma}_{j}, \hat{\sigma}_{l}, t\right)\right],
\end{aligned}
$$

where the redistribution mechanism

$$
\begin{gathered}
W_{j l}\left(\sigma_{j} \sigma_{l} \rightarrow \hat{\sigma}_{j} \hat{\sigma}_{l}\right)=\frac{1}{Q_{j l}} \delta_{\sigma_{j}+\sigma_{l}, \hat{\sigma}_{j}+\hat{\sigma}_{l}} \exp \left[-\beta H\left(\left\{\sigma_{m}\right\}_{m \neq j, l}, \hat{\sigma}_{j}, \hat{\sigma}_{l}\right)\right], \\
Q_{j l}=\sum_{\hat{\sigma}_{j} \hat{\sigma}_{l}} \delta_{\sigma_{j}+\sigma_{l}, \hat{\sigma}_{j}+\hat{\sigma}_{l}} \exp \left[-\beta H\left(\left\{\sigma_{m}\right\}_{m \neq j, l}, \hat{\sigma}_{j}, \hat{\sigma}_{l}\right)\right] .
\end{gathered}
$$

With the master equation above, we can further get that

$$
\begin{aligned}
\frac{d}{d t} q_{k}(t)= & Q_{k}^{K}=-2 D q_{k}(t)+\sum_{\{\sigma\}} \sum_{\omega}\left[\sum_{\hat{\sigma}_{k}, \hat{\sigma}_{k+\omega}} \hat{\sigma}_{k} W_{k, k+\omega}\left(\sigma_{k} \sigma_{k+\omega} \rightarrow \hat{\sigma}_{k} \hat{\sigma}_{k+\omega}\right)\right] P(\{\sigma\}, t) \\
& +p_{A}\left\{-(N-1) q_{k}(t)+\sum_{\{\sigma\}}\left[\sum_{l \neq k} \sum_{\hat{\sigma}_{k}, \hat{\sigma}_{l}} \hat{\sigma}_{k} W_{k l}\left(\sigma_{k} \sigma_{l} \rightarrow \hat{\sigma}_{k} \hat{\sigma}_{l}\right)\right] P(\{\sigma\}, t)\right\},
\end{aligned}
$$

where $\sum_{\omega}$ denotes the summation taken over nearest neighbors.

\section{Competing mechanism}

Naturally, both Glauber's dynamics and Kawasaki's dynamics favor a lower energy state. However, when the system under study is in contact with a heat bath while exposed to an external energy flux, one requires a competition between a process that favors lower system energy and another process that favors higher system energy. Usually, Glauber's mechanism is used to simulate the contact of the system with a heat bath and favors a lower energy state. Meanwhile Kawasaki's mechanism can be modified in order to simulate an external energy flux that drives the system towards higher energy. This can be achieved by switching $\beta$ to $-\beta$, or $K=\beta J=J / K_{B} T$ to $-K$, and modifying the redistribution probability accordingly, i.e.

$$
W_{j l} \propto \exp [-\beta H] \Rightarrow W_{j l} \propto \exp [+\beta H]
$$

This means that the competition between Glauber's mechanism and Kawasaki's mechanism is actually that between ferromagnetism and antiferromagnetism.

Based on the above considerations, we use the competing mechanism to simulate the dynamics of a system in contact with a heat bath and exposed to an external energy flux simultaneously. The master equation can be written as (Eq. (1)),

$$
\frac{d}{d t} P(\{\sigma\}, t)=p G_{m e}+(1-p) K_{m e}
$$

where

$$
G_{m e}=-\sum_{i} \sum_{\sigma}\left[W_{i}\left(\sigma_{i} \rightarrow \hat{\sigma}_{i}\right) P(\{\sigma\}, t)-W_{i}\left(\hat{\sigma}_{i} \rightarrow \sigma_{i}\right) P\left(\left\{\sigma_{j \neq i}\right\}, \hat{\sigma}, t\right) .\right.
$$


and

$$
\begin{aligned}
K_{m e}= & \sum_{\langle j l\rangle} \sum_{\hat{\sigma}_{j} \hat{\sigma}_{l}}\left[-W_{j l}\left(\sigma_{j} \sigma_{l} \rightarrow \hat{\sigma}_{j} \hat{\sigma}_{l}\right) P(\{\sigma\}, t)\right. \\
& \left.+W_{j l}\left(\hat{\sigma}_{j} \hat{\sigma}_{l} \rightarrow \sigma_{j} \sigma_{l}\right) P\left(\left\{\sigma_{j \neq i}, \sigma_{j \neq i}\right\}, \hat{\sigma}_{j}, \hat{\sigma}_{l}, t\right)\right] \\
& +\frac{1}{2} p_{A} \sum_{j} \sum_{l \neq j} \sum_{\hat{\sigma}_{j} \hat{\sigma}_{l}}\left[-W_{j l}\left(\sigma_{j} \sigma_{l} \rightarrow \hat{\sigma}_{j} \hat{\sigma}_{l}\right) P(\{\sigma\}, t)\right. \\
& \left.+W_{j l}\left(\hat{\sigma}_{j} \hat{\sigma}_{l} \rightarrow \sigma_{j} \sigma_{l}\right) P\left(\left\{\sigma_{j \neq i}, \sigma_{l \neq k}\right\}, \hat{\sigma}_{j}, \hat{\sigma}_{l}, t\right)\right] .
\end{aligned}
$$

From the master equation, we can obtain the evolving equation of single spins,

$$
\frac{d}{d t} q_{k}(t)=p Q_{k}^{G}+(1-p) Q_{k}^{K} .
$$

The first term describes the Glauber-type dynamics which is used to simulate the influence of the energy flux and $Q_{k}^{G}$ is given by Eq. (3). The second term describes the Kawasaki-type dynamics which is used to simulate the influence of the energy flux. As mentioned above, we require it to favor higher energy state. However, the redistribution probability, Eq. (5), clearly favors a lower energy state. Considering this, we can use the expression of Eq. (6) for $Q_{k}^{K}$, if we simply switch the sign before $\beta$ in the redistribution probability.

\section{The competing dynamics in the Gaussian model}

Now we turn to the Gaussian model built on an A-SWN, of which the Hamiltonian can be written as

$$
\begin{aligned}
-\beta \mathcal{H}(\{\sigma\})= & K \sum_{i j k} \sigma_{i j k}\left(\sigma_{i+1, j, k}+\sigma_{i, j+1, k}+\sigma_{i, j, k+1}\right) \\
& +\frac{1}{2} p_{A} K \sum_{i j k} \sigma_{i j k} \sum_{i^{\prime} j^{\prime} k^{\prime}} \sigma_{i^{\prime} j^{\prime} k^{\prime}}-\frac{1}{2} p_{A} K \sum_{i j k} \sigma_{i j k}^{2},
\end{aligned}
$$

where $\beta=1 / k_{B} T$ and $K=\beta J$. We only discuss the case of $K>0$, namely, $J>0$. This case corresponds to the ferromagnetic system. The expression (9) is obtained by taking into consideration the fact that the influence of the system as a whole on individual spins is of the mean-field nature [10] (for related discussions see Ref. [4-9]). The spins can take any real value from $-\infty$ to $+\infty$. The probability of finding a given spin between $\sigma_{k}$ and $\sigma_{k}+d \sigma_{k}$ is assumed to be the Gaussian-type distribution, $f\left(\sigma_{k}\right) d \sigma_{k}=\sqrt{\frac{b}{2 \pi}} \exp \left(-\frac{b}{2} \sigma_{k}^{2}\right) d \sigma_{k}$, where $b$ is a distribution constant independent of temperature. Therefore, the summation of the spin value turns into an integration, and we can further obtain from Eqs. (3) and (6),

$$
Q_{i j k}^{G}=-q_{i j k}(t)+\frac{K}{b} \sum_{\omega= \pm 1}\left(q_{i+\omega, j, k}(t)+q_{i, j+\omega, k}(t)+q_{i, j, k+\omega}(t)\right)+\frac{K}{b}(N-1) p_{A} M(t),(K>0)
$$

where $M(t) \equiv \frac{1}{N} \sum_{k} q_{k}(t)$, and

$$
\begin{aligned}
Q_{i j k}^{K}= & \frac{1}{2[b+(-K)]} b\left\{\left[\left(q_{i+1, j, k}-q_{i, j, k}\right)-\left(q_{i, j, k}-q_{i-1, j, k}\right)\right]\right. \\
& \left.+\left[\left(q_{i, j+1, k}-q_{i, j, k}\right)-\left(q_{i, j, k}-q_{i, j-1, k}\right)\right]+\left[\left(q_{i, j, k+1}-q_{i, j, k}\right)-\left(q_{i, j, k}-q_{i, j, k-1}\right)\right]\right\} \\
& +\frac{(-K)}{2[b+(-K)]}\left[2\left(2 q_{i-1, j, k}-q_{i-1, j+1, k}-q_{i-1, j-1, k}\right)+\left(2 q_{i-1, j, k}-q_{i, j, k}-q_{i-2, j, k}\right)\right. \\
& +2\left(2 q_{i+1, j, k}-q_{i+1, j+1, k}-q_{i+1, j-1, k}\right)+\left(2 q_{i+1, j, k}-q_{i, j, k}-q_{i+2, j, k}\right) \\
& +2\left(2 q_{i, j-1, k}-q_{i, j-1, k+1}-q_{i, j-1, k-1}\right)+\left(2 q_{i, j-1, k}-q_{i, j, k}-q_{i, j-2, k}\right) \\
& +2\left(2 q_{i, j+1, k}-q_{i, j+1, k+1}-q_{i, j+1, k-1}\right)+\left(2 q_{i, j+1, k}-q_{i, j, k}-q_{i, j+2, k}\right) \\
& +2\left(2 q_{i, j, k-1}-q_{i+1, j, k-1}-q_{i,-1 j, k-1}\right)+\left(2 q_{i, j,-1 k}-q_{i, j, k}-q_{i, j-2, k}\right) \\
& \left.+2\left(2 q_{i, j, k+1}-q_{i+1, j, k+1}-q_{i-1, j, k+1}\right)+\left(2 q_{i, j, k+1}-q_{i, j, k}-q_{i, j, k+2}\right)\right] \\
& -\frac{p_{A}}{2}(N-1)\left[q_{i j k}(t)-M(t)\right]+p_{A}(N-1) \frac{(-K)}{2 b} \\
& \times \sum_{\omega= \pm 1}\left[\left(q_{i+\omega, j, k}-M(t)\right)+\left(q_{i, j+\omega, k}-M(t)\right)+\left(q_{i, j, k+\omega}-M(t)\right], \quad(b>K>0)\right.
\end{aligned}
$$


where $b>K>0$ is required by the convergence of the integration.

Now we determine the system behavior by studying the following order parameters. First, from Eq. (8) we obtain

$$
\begin{aligned}
\frac{d}{d t} M(t) & \equiv \frac{1}{N} \sum_{i j k} \frac{d}{d t} q_{i j k}(t)=p \frac{1}{N} \sum_{i j k} Q_{i j k}^{G}+(1-p) \frac{1}{N} \sum_{i j k} Q_{i j k}^{K} \\
& =-p\left[1-\frac{6 K}{b}-\frac{K}{b}(N-1) p_{A}\right] M(t),
\end{aligned}
$$

$\sum_{i j k} Q_{i j k}^{K}=0$ means that the Kawasaki-type dynamics does not change the value of $M(t)$. Second, we define

$$
M^{\prime}(t) \equiv \frac{1}{N} \sum_{i j k} q_{i j k}^{\prime}(t) \equiv \frac{1}{N} \sum_{i j k}(-1)^{i+j+k} q_{i j k}(t),
$$

and similarly we obtain

$$
\frac{d}{d t} M^{\prime}(t) \equiv \frac{1}{N} \sum_{i j k} \frac{d}{d t} q_{i j k}^{\prime}(t)=p \frac{1}{N} \sum_{i j k} Q_{i j k}^{\prime G}+(1-p) \frac{1}{N} \sum_{i j k} Q_{i j k}^{\prime K},
$$

where

$$
Q_{i j k}^{\prime G}=-q_{i j k}^{\prime}(t)-\frac{K}{b} \sum_{\omega= \pm 1}\left[q_{i+\omega, j, k}^{\prime}(t)+q_{i, j+\omega, k}^{\prime}(t)+q_{i, j, k+\omega}^{\prime}(t)\right]+\frac{K}{b}(N-1) p_{A}(-1)^{i+j+k} M(t)
$$

and

$$
\begin{aligned}
Q_{i j k}^{\prime K}= & \frac{1}{2(b-K)} b\left\{\left[\left(-q_{i+1, j, k}^{\prime}-q_{i, j, k}^{\prime}\right)-\left(q_{i, j, k}^{\prime}+q_{i-1, j, k}^{\prime}\right)\right]\right. \\
& \left.+\left[\left(-q_{i, j+1, k}^{\prime}-q_{i, j, k}^{\prime}\right)-\left(q_{i, j, k}^{\prime}+q_{i, j-1, k}^{\prime}\right)\right]+\left[\left(-q_{i, j, k+1}^{\prime}-q_{i, j, k}^{\prime}\right)-\left(q_{i, j, k}^{\prime}+q_{i, j, k-1}^{\prime}\right)\right]\right\} \\
& -\frac{K}{2(b-K)}\left[2\left(-2 q_{i-1, j, k}^{\prime}-q_{i-1, j+1, k}^{\prime}-q_{i-1, j-1, k}^{\prime}\right)+\left(-2 q_{i-1, j, k}^{\prime}-q_{i, j, k}^{\prime}-q_{i-2, j, k}^{\prime}\right)\right. \\
& +2\left(-2 q_{i+1, j, k}^{\prime}-q_{i+1, j+1, k}^{\prime}-q_{i+1, j-1, k}^{\prime}\right)+\left(-2 q_{i+1, j, k}^{\prime}-q_{i, j, k}^{\prime}-q_{i+2, j, k}^{\prime}\right) \\
& +2\left(-2 q_{i, j-1, k}^{\prime}-q_{i, j-1, k+1}^{\prime}-q_{i, j-1, k-1}^{\prime}\right)+\left(-2 q_{i, j-1, k}^{\prime}-q_{i, j, k}^{\prime}-q_{i, j-2, k}^{\prime}\right) \\
& +2\left(-2 q_{i, j+1, k}^{\prime}-q_{i, j+1, k+1}^{\prime}-q_{i, j+1, k-1}^{\prime}\right)+\left(-2 q_{i, j+1, k}^{\prime}-q_{i, j, k}^{\prime}-q_{i, j+2, k}^{\prime}\right) \\
& +2\left(-2 q_{i, j, k-1}^{\prime}-q_{i+1, j, k-1}^{\prime}-q_{i,-1 j, k-1}\right)+\left(-2 q_{i, j,-1 k}^{\prime}-q_{i, j, k}^{\prime}-q_{i, j-2, k}^{\prime}\right) \\
& \left.+2\left(-2 q_{i, j, k+1}^{\prime}-q_{i+1, j, k+1}^{\prime}-q_{i-1, j, k+1}^{\prime}\right)+\left(-2 q_{i, j, k+1}^{\prime}-q_{i, j, k}^{\prime}-q_{i, j, k+2}^{\prime}\right)\right] \\
& -\frac{p_{A}(N-1)}{2}\left\{q_{i j k}^{\prime}(t)-(-1)^{i+j+k} M(t)\right. \\
& \left.-\frac{K}{b} \sum_{\omega}\left(q_{i+\omega, j, k}^{\prime}(t)+q_{i, j+\omega, k}^{\prime}(t)+q_{i, j, k+\omega}^{\prime}(t)\right)-\frac{6 k}{b}(-1)^{i+j+k} M(t)\right\} .
\end{aligned}
$$

Performing the summation over the indices $i, j$, and $k$, we get

$$
\frac{d}{d t} M^{\prime}(t)=\left\{-p\left(1+\frac{6 K}{b}\right)+(1-p)\left[6 \frac{6 K-b}{b-K}-\frac{p_{A}(N-1)}{2}\left(1-\frac{6 K}{b}\right)\right]\right\} M^{\prime}(t) .
$$

The Solutions of equations (11) and (13) are

$$
M(t)=M(0) \exp \left\{-p\left[1-\left(1+\frac{(N-1) p_{A}}{6}\right) \frac{K}{K_{c}^{0}}\right] t\right\},(K>0)
$$

and

$$
\begin{aligned}
M^{\prime}(t)= & M^{\prime}(0) \exp \left\{-\left(p\left(1+\frac{K}{K_{c}^{0}}\right)+(1-p)\left[\frac{36}{6-K / K_{c}^{0}}+\frac{p_{A}(N-1)}{2}\right]\left(1-\frac{K}{K_{c}^{0}}\right)\right) t\right\}, \\
& \left(\begin{array}{c}
\text { if } p=1, \text { then } K>0 \\
\text { if } p \neq 1, \text { then }(b>K>0)
\end{array}\right)
\end{aligned}
$$


where $K_{c}^{0}=|J| / k_{B} T_{c}=b / 6$ is the critical point of the three-dimensional Gaussian model without the SWN effect.

(1) When the SWN effect does not exist and $p_{A}=0$,

$$
\begin{gathered}
M(t)=M(0) \exp \left[-p\left(1-K / K_{c}^{0}\right) t\right] \\
M^{\prime}(t)=M^{\prime}(0) \exp \left\{-\left[p\left(1+K / K_{c}^{0}\right)+36(1-p) \frac{1-K / K_{c}^{0}}{6-K / K_{c}^{0}}\right] t\right\} .
\end{gathered}
$$

Analyzing the long-time system behavior, we find that:

(1.a) For the case of

$$
K<K_{c}^{0}, \quad\left(T>T_{c}^{0}\right),
$$

we have both vanishing $M(t)$ and $M^{\prime}(t)$, which correspond to the paramagnetic phase.

(1.b) For the case of

$$
K \rightarrow K_{c}^{0}
$$

$M^{\prime}(t) \rightarrow 0$, and

$$
M(t)=M(0) \exp \left[-p \frac{t}{\tau}\right], \tau=\frac{1}{1-K / K_{c}^{0}} \rightarrow \infty .
$$

The critical slowing down of the order parameter $M(t)$ will appear at the critical point $K_{c}^{0}$.

(1.c) For the case of

$$
K>K_{c}^{0}, \quad\left(T<T_{c}\right),
$$

and

$$
\frac{K}{K_{c}^{0}}<\frac{1}{2 p}\left(-36+41 p+\sqrt{\left(1561 p^{2}-2808 p+1296\right)}\right)
$$

we have nonvanishing $M(t)$ and vanishing $M^{\prime}(t)$, which correspond to the ferromagnetic phase.

(1.d) For the case of

$$
\frac{1}{2 p}\left(-36+41 p+\sqrt{\left(1561 p^{2}-2808 p+1296\right)}\right)<\frac{K}{K_{c}^{0}}<6
$$

we have both nonvanishing $M(t)$ and $M^{\prime}(t)$.

With both of the order parameters nonvanishing, this phase cannot be simply identified as ferromagnetic or antiferromagnetic. We name it as heterophase, and from Eqs. (16) and (17) we can see that the system behavior strongly depends on the initial condition and the temperature.

(1.e) For the case of

$$
K / K_{c}^{0} \geq 6,
$$

we could obtain $M(t) \neq 0$, and when $p=1$ (with pure Glauber-type dynamics simulating the contact with a heat bath), we have $M^{\prime}(t) \rightarrow 0$. However, with $p \neq 1$ (with an external energy flow), because the antiferromagnetic Kawasaki-type dynamics is limited by the condition $(b>K>0)$ to ensure the convergence of the integration, we cannot obtain the value of $M^{\prime}(t)$. As a result, we cannot theoretically obtain the system behavior in the temperature region $K / K_{c}^{0} \geq 6^{1}$.

The phase diagram is shown in Fig. 1(a).

(2) Now the A-SWN effect is introduced and $p_{A} \neq 0$. We suppose $p_{A}=1 / N$, and then we have

\footnotetext{
${ }^{1}$ The same problem exists in Ref. [19], i.e., the system behavior cannot be theoretically obtained for the region $K \geq b$.
} 


$$
M(t)=M(0) \exp \left\{-p\left[1-\left(1+\frac{1}{6}\right) \frac{K}{K_{c}^{0}}\right] t\right\}
$$

and

$$
M^{\prime}(t)=M^{\prime}(0) \exp \left\{-\left(p\left(1+K / K_{c}\right)+(1-p)\left[\frac{36}{6-K / K_{c}^{0}}+\frac{1}{2}\right]\left(1-\frac{K}{K_{c}^{0}}\right)\right) t\right\} .
$$

Analyzing the long-time asymptotic behavior, we can similarly get:

(2.a) For the case of

$$
K<\left.K_{c}\right|_{p_{A}=1 / N}=\frac{6}{7} K_{c}^{0}
$$

the condition leads to vanishing $M(t)$ and vanishing $M^{\prime}(t)$, which correspond to the paramagnetic phase.

(2.b) For the case of

$$
\left.K \rightarrow K_{c}\right|_{p_{A}=1 / N}=\frac{6}{7} K_{c}^{0}
$$

The critical slowing down of the system will appear.

(2.c) For the case of

$$
\frac{89 p-79+\sqrt{7129 p^{2}-12862 p+5929}}{2(3 p-1)}>\frac{K}{K_{c}^{0}}>\frac{6}{7}
$$

we have nonvanishing $M(t)$ and vanishing $M^{\prime}(t)$, which correspond to the ferromagnetic phase.

(2.d) For the case of

$$
6>\frac{K}{K_{c}^{0}}>\frac{89 p-79+\sqrt{7129 p^{2}-12862 p+5929}}{2(3 p-1)}
$$

we have both nonvanishing $M(t)$ and $M^{\prime}(t)$, which correspond to the heterophase.

(2.e) For the case of

$$
K / K_{c}^{0} \geq 6
$$

For the same reason (see (1.e)), we cannot obtain theoretically the system behavior.

The phase diagram is shown in Fig. 1(b).

\section{COMPETING MECHANISM ON R-SWN}

Now we investigate the competing dynamics on rewiring-type small-world networks (the definition see the Introduction), and the sequence of the content is the same as that in the previous section.

With the Glauber-type mechanism, the master equation has the same form as Eq. (2), and the single-spin evolving equation is also given by Eq. (3).

With the Kawasaki-type mechanism, the master equation can be written as

$$
\begin{aligned}
\frac{d}{d t} P(\{\sigma\}, t)= & \left(1-p_{R}\right) \sum_{\langle j l\rangle} \sum_{\hat{\sigma}_{j} \hat{\sigma}_{l}}\left[-W_{j l}\left(\sigma_{j} \sigma_{l} \rightarrow \hat{\sigma}_{j} \hat{\sigma}_{l}\right) P(\{\sigma\}, t)\right. \\
& \left.+W_{j l}\left(\hat{\sigma}_{j} \hat{\sigma}_{l} \rightarrow \sigma_{j} \sigma_{l}\right) P\left(\left\{\sigma_{j \neq i}, \sigma_{l \neq k}\right\}, \hat{\sigma}_{j}, \hat{\sigma}_{l}, t\right)\right] \\
& +\frac{D p_{R}}{N-1} \sum_{j} \sum_{l \neq j} \sum_{\hat{\sigma}_{j} \hat{\sigma}_{l}}\left[-W_{j l}\left(\sigma_{j} \sigma_{l} \rightarrow \hat{\sigma}_{j} \hat{\sigma}_{l}\right) P(\{\sigma\}, t)\right. \\
& \left.+W_{j l}\left(\hat{\sigma}_{j} \hat{\sigma}_{l} \rightarrow \sigma_{j} \sigma_{l}\right) P\left(\left\{\sigma_{j \neq i}, \sigma_{l \neq k}\right\}, \hat{\sigma}_{j}, \hat{\sigma}_{l}, t\right)\right] .
\end{aligned}
$$

The redistribution probability $W_{j l}$ is given by Eq. (5), and the single-spin evolving equation 


$$
\begin{aligned}
\frac{d}{d t} q_{k}(t)= & Q_{k}^{K}=\left(1-p_{R}\right)\left\{-2 D q_{k}(t)+\sum_{\{\sigma\}} \sum_{\omega= \pm 1}\left[\sum_{\hat{\sigma}_{k}, \hat{\sigma}_{k+\omega}} \hat{\sigma}_{k} W_{k, k+\omega}\left(\sigma_{k} \sigma_{k+w} \rightarrow \hat{\sigma}_{k} \hat{\sigma}_{k+w}\right)\right] P(\{\sigma\}, t)\right\} \\
& +\frac{D p_{R}}{N-1}\left\{-(N-1) q_{k}(t)+\sum_{\{\sigma\}}\left[\sum_{l \neq k} \sum_{\hat{\sigma}_{k}, \hat{\sigma}_{l}} \hat{\sigma}_{k} W_{k l}\left(\sigma_{k} \sigma_{l} \rightarrow \hat{\sigma}_{k} \hat{\sigma}_{l}\right)\right] P(\{\sigma\}, t)\right\} .
\end{aligned}
$$

With the competing mechanism, the evolution of $q_{k}(t)$ is once again described by an equation having the same form as Eq. (8)

$$
\frac{d}{d t} q_{k}(t)=p Q_{k}^{G}+(1-p) Q_{k}^{K}
$$

which consists of two terms, one corresponding to the Glauber-type dynamics and the other corresponding to the Kawasaki-type dynamics. In the following we turn to the Gaussian model.

For the three-dimensional Gaussian model built on a R-SWN, the Hamiltonian can be written as

$$
\begin{aligned}
-\beta H= & K\left(1-p_{R}\right) \sum_{i j k} \sigma_{i j k}\left(\sigma_{i+1, j, k}+\sigma_{i, j+1, k}+\sigma_{i, j, k+1}\right) \\
& +\frac{3}{N} p_{R} K \sum_{i j k} \sigma_{i j k} \sum_{i^{\prime} j^{\prime} k^{\prime}} \sigma_{i^{\prime} j^{\prime} k^{\prime}}-\frac{3}{N} p_{R} K \sum_{i j k} \sigma_{i j k}^{2} .
\end{aligned}
$$

In the single-spin evolving equation with the competing mechanism, the term that corresponds to the Glauber-type dynamics is now given by

$$
Q_{i j k}^{G}=-q_{i j k}(t)+\frac{K}{b}\left(1-p_{R}\right) \sum_{\omega= \pm 1}\left(q_{i+\omega, j, k}+q_{i, j+\omega, k}+q_{i, j, k+\omega}\right)+\frac{6 p_{R}}{b} K M(t),(K>0)
$$

and the term that corresponds to the Kawasaki-type dynamics can be written as (similarly, we switch $\beta$ to $-\beta$, and $K$ to $-K)$

$$
\begin{aligned}
Q_{i j k}^{K}= & \frac{1}{2\left[b-K\left(1-p_{R}\right)\right]} b\left\{\left[\left(q_{i+1, j, k}-q_{i, j, k}\right)-\left(q_{i, j, k}-q_{i-1, j, k}\right)\right]\right. \\
& \left.+\left[\left(q_{i, j+1, k}-q_{i, j, k}\right)-\left(q_{i, j, k}-q_{i, j-1, k}\right)\right]+\left[\left(q_{i, j, k+1}-q_{i, j, k}\right)-\left(q_{i, j, k}-q_{i, j, k-1}\right)\right]\right\} \\
& -\frac{K\left(1-p_{R}\right)}{2\left[b-K\left(1-p_{R}\right)\right]}\left[2\left(2 q_{i-1, j, k}-q_{i-1, j+1, k}-q_{i-1, j-1, k}\right)+\left(2 q_{i-1, j, k}-q_{i, j, k}-q_{i-2, j, k}\right)\right. \\
& +2\left(2 q_{i+1, j, k}-q_{i+1, j+1, k}-q_{i+1, j-1, k}\right)+\left(2 q_{i+1, j, k}-q_{i, j, k}-q_{i+2, j, k}\right) \\
& +2\left(2 q_{i, j-1, k}-q_{i, j-1, k+1}-q_{i, j-1, k-1}\right)+\left(2 q_{i, j-1, k}-q_{i, j, k}-q_{i, j-2, k}\right) \\
& +2\left(2 q_{i, j+1, k}-q_{i, j+1, k+1}-q_{i, j+1, k-1}\right)+\left(2 q_{i, j+1, k}-q_{i, j, k}-q_{i, j+2, k}\right) \\
& +2\left(2 q_{i, j, k-1}-q_{i+1, j, k-1}-q_{i,-1 j, k-1}\right)+\left(2 q_{i, j,-1 k}-q_{i, j, k}-q_{i, j-2, k}\right) \\
& +2\left(2 q_{i, j, k+1}-q_{i+1, j, k+1}-q_{i-1, j, k+1}\right)+\left(2 q_{i, j, k+1}-q_{i, j, k}-q_{i, j, k+2}\right) \\
& -\frac{3}{2} p_{R}\left\{q_{i j k}(t)-M(t)+\frac{K\left(1-p_{R}\right)}{b}\left[\sum_{\omega= \pm 1}\left(q_{i+\omega, j, k}+q_{i, j+\omega, k}+q_{i, j, k+\omega}\right)-6 M(t)\right]\right\}, \\
& \left(b>K\left(1-p_{R}\right)>0\right)
\end{aligned}
$$

where $b>K\left(1-p_{R}\right)>0$ is required by the convergency of the integration.

From the single-spin evolving equation with the competing mechanism, we can obtain

$$
\frac{d}{d t} M(t)=\frac{1}{N} \sum_{i j k} \frac{d}{d t} q_{i j k}(t)=p\left(-1+\frac{6 K}{b}\right) M(t),
$$

and 


$$
\begin{aligned}
\frac{d}{d t} M^{\prime}(t)= & p \frac{1}{N} \sum_{i j K} Q_{i j k}^{\prime G}+(1-p) \frac{1}{N} \sum_{i j k} Q_{i j k}^{\prime K} \\
= & \left(-p\left[1+\frac{6 K}{b}\left(1-p_{R}\right)\right]\right. \\
& \left.+(1-p)\left\{6 \frac{6 K\left(1-p_{R}\right)-b}{b-K\left(1-p_{R}\right)}-\frac{3 p_{R}}{2}\left[1-\frac{6 K}{b}\left(1-p_{R}\right)\right]\right\}\right) M^{\prime}(t) .
\end{aligned}
$$

The solutions of Eqs. (23) and (24) are, respectively,

$$
M(t)=M(0) \exp \left[-p\left(1-K / K_{c}^{0}\right) t\right],(K>0)
$$

and

$$
\begin{aligned}
M^{\prime}(t)= & M^{\prime}(0) \exp \left(-\left\{p\left[1+\left(1-p_{R}\right) \frac{K}{K_{c}^{0}}\right]\right.\right. \\
& \left.\left.+(1-p)\left[\frac{36}{6-K\left(1-p_{R}\right) / K_{c}^{0}}+\frac{3 p_{R}}{2}\right]\left[1-\left(1-p_{R}\right) \frac{K}{K_{c}^{0}}\right]\right\}\right), \\
& \left(\begin{array}{c}
\text { if } p=1, \text { then } K>0 \\
\text { if } p \neq 1, \text { then }\left(b>K\left(1-p_{R}\right)>0\right)
\end{array}\right),
\end{aligned}
$$

where $K_{c}^{0}=J / k_{B} T_{c}^{0}=b / 6$ is the critical point without the SWN effect.

(1) When no SWN effect is considered and $p_{R}=0$, the phase diagram is given by Fig. 1 (a).

(2) Now we introduce the R-SWN effect and set $p_{R}=0.1$, and then Eqs. (25) and (26) become

$$
M(t)=M(0) \exp \left[-p\left(1-K / K_{c}^{0}\right) t\right],
$$

and

$$
M^{\prime}(t)=M^{\prime}(0) \exp \left\{-\left[p\left(1+\frac{9 K}{10 K_{c}^{0}}\right)+(1-p)\left(\frac{36}{6-\frac{9 K}{10 K_{c}^{0}}}+\frac{3}{20}\right)\left(1-\frac{9 K}{10 K_{c}^{0}}\right)\right]\right\} .
$$

Analyzing the long-time asymptotic behavior, we find that:

(2.a) For the case of

$$
K<K_{c}^{0}, \quad\left(T>T_{c}^{0}\right),
$$

we have both vanishing $M(t)$ and $M^{\prime}(t)$, and this corresponds to the paramagnetic phase.

(2.b) For the case of

$$
K \rightarrow K_{c}^{0},
$$

we shall observe the critical slowing down of the system.

(2.c) For the case of

$$
K>K_{c}^{0},\left(T<T_{c}^{0}\right)
$$

and

$$
\frac{K}{K_{c}^{0}}<\frac{25230 p-22230+150 \sqrt{26017 p^{2}-46842 p+21609}}{2(621 p-81)},
$$

the condition leads to nonvanishing $M(t)$ and vanishing $M^{\prime}(t)$, which correspond to the ferromagnetic phase.

(2.d) For the case of

$$
\frac{25230 p-22230+150 \sqrt{26017 p^{2}-46842 p+21609}}{2(621 p-81)}<\frac{K}{K_{c}^{0}}<\frac{20}{3},
$$

we have both nonvanishing $M(t)$ and $M^{\prime}(t)$, which correspond to the heterophase.

(2.e) For the case of

$$
K / K_{c}^{0} \geq 20 / 3
$$

For the same reason (see Sec. II. (1.e)), we cannot give theoretically the behavior of the system in this region.

The phase diagram is shown in Fig. 1(c). 


\section{SUMMARY}

Comparing Fig. 1(a), (b) and (c), we find both similarities and differences. The phase diagrams have similar structures while the boundaries may be shifted by the SWN effect. In the following we briefly explain our observations and discuss the nature of the competing mechanism and the SWN effect.

First we notice that the phase diagrams are all separated into four regions, with two special temperatures: one is the critical temperature $K_{c}=J / k_{B} T_{c}$, another is the limit temperature $K_{\max }=J / k_{B} T_{\min }$ required by the convergence of the integration. When the SWN effect does not exist, $K_{c}=K_{c}^{0}=J / k_{B} T_{c}^{0}=b / 2 D$ is the critical point of the Gaussian model, where $b$ is the Gaussian distribution constant and $D$ is the space dimension, and $K_{\max }=b$ (since $b>K=|J| / k_{B} T>0$ is the region where we can assure the convergence of the integration in the Kawasakitype dynamics of the antiferromagnetic system). When the A-SWN effect is introduced, $K_{c}=\frac{6}{6+(N-1) p_{A}} K_{c}^{0}$ and $K_{\max }=b=2 D K_{c}^{0}$ (in the example studied $D=3, p_{A} \approx 1 / N$, and $K_{c} / K_{c}^{0}=6 / 7$ ). When the R-SWN effect is introduced, $K_{c}=K_{c}^{0}$ and $K_{\max }=\frac{b}{\left(1-p_{R}\right)}=\frac{2 D K_{c}^{0}}{\left(1-p_{R}\right)}$ (in the example studied $D=3, p_{R}=0.1$, and $K_{\max } / K_{c}^{0}=20 / 3$ ). Now we describe the phase behavior: Above the critical temperature $T_{c}=J / k_{B} K_{c}$, one can only observe a disordered state, namely the paramagnetic phase, because of the dominating heat noise. Below critical temperature $T_{c}$, the system begins to show some kind of order, and the system behavior is determined by the competition between the Glauber-type mechanism which favors a ferromagnetic state, and the Kawasaki-type mechanism which favors an antiferromagnetic state. The result of the competition is subject to two factors, the probability of each mechanism and the temperature. However, the Kawasaki-type dynamics describing an antiferromagnetic Gaussian system is limited by the condition $T>T_{\min }$, which is necessary for the convergence of the integration. As a result, in the region $T \leq T_{\min }$ we cannot theoretically obtain the system behavior.

Now we discuss how and why the two special temperatures $T_{c}$ and $T_{\min }$ may be affected by the SWN effect. When the Gaussian model is built on a R-SWN, the temperature $T_{c}\left(=T_{c}^{0}\right)$ remains the same. This is because, as is clear in all of the phase diagrams, $T_{c}$ is determined by the competition between the heat noise and the Glauber-type mechanism, which favors a lower energy state. One important characteristic of the Gaussian model is that this temperature can be further determined by the average coordination number. For example, without the SWN effect this temperature is given by $J / k_{B} T_{c}=b / 2 D$, where $2 D$ is the average coordination number of a $D$-dimensional lattice. On a R-SWN, the average coordination number is not changed when a portion of the regular links are rewired, and thus $T_{c}$ also remains unchanged. On the other hand the temperature $T_{\min }$ is lower than the value on a regular network. On R-SWN long range links have made long range spin-pair redistribution possible, and therefore the influence of the Kawasaki-type mechanism is enhanced. Thus it is not difficult to understand why on R-SWN there is a lower value of the temperature $T_{\min }$.

When the Gaussian model is built on an A-SWN, the temperature $T_{c}\left(=\frac{6+(N-1) p_{A}}{6} T_{c}^{0}\right)$ becomes higher. As is explained above, this temperature is determined by the average coordination number, which is $\frac{7}{6} \cdot 2 D$ on a three dimensional A-SWN with $p_{A} \approx 1 / N$. At the same time the temperature $T_{\min }$ is unchanged. We may explain that it is because the two dynamics are both strengthened, i.e., by the larger coordination number and the long range spin-pair redistribution, and the temperature that characterizes a certain counterbalance remains the same.

To summarize, in this article we investigate the competing Glauber-type and Kawasaki-type dynamics on two typical three-dimensional small-world networks, adding-type (A-SWN) and rewiring -type (R-SWN), in the framework of the Gaussian model. We get the evolution of the order parameters, $M(t)$ and $M^{\prime}(t)$, and by analyzing the long time asymptotic behavior we draw the phase diagrams. With the competing mechanism, there exist two easily identifiable special temperatures. The influence of the long range links is analyzed: For the Glauber-type dynamics, more important is the altered average coordination number while the Kawasaki-type dynamics is enhanced by the long range spin interaction and redistribution.

\section{ACKNOWLEDGMENTS}

This work was supported by the National Natural Science Foundation of China under Grant No. 10075025.

[1] D. J. Watts and S. H Strogatz, Nature 393, 440 (1998). 
[2] S. H Strogatz, Nature 410, 268 (2001).

[3] Reka Albert and Albert-Laszlo Barabasi, Rev. Mod. Phys. 74, 47 (2002).

[4] M. E. J. Newman, SIAM Review 45, 167 (2003).

[5] A. Barrat and M. Weigt, Eur. Phys. J. B 13, 547 (2000).

[6] A. Pekalski, Phys. Rev. E 64, 057104 (2001).

[7] C. P. Herrero, Phys Rev. E 65, 066110 (2002).

[8] H. Hong, B. J. Kim, and M. Y. Choi, Phys. Rev. E 66, 018101 (2002).

[9] B. J. Kim, H. Hong, P. Holme, G. S. Jeon, P. Minnhagen, and M. Y. Choi, Phys. Rev. E 64, 056135 (2001).

[10] Jian-Yang Zhu and Han Zhu, Phys. Rev. E 67, 026125 (2003).

[11] R. J. Glauber, J. Math. Phys. 4, 294 (1963).

[12] Kawasaki, Phys. Rev. 145, 224 (1965).

[13] T. Tome and M. J. de Oliveira, Phys. Rev. A 40, 6643 (1989).

[14] B. C. S. Grandi and W. Figueiredo, Phys. Rev. E 53, 5484 (1996); 54, 4722 (1996); 56, 5240 (1996); Phys. Rev. B 50, 12595 (1994).

[15] Ma Yuqiang and Liu Jiwen, Phys. Lett. A 238, 159 (1998).

[16] J. R. S. Leao, B. C. S. Grandi, and W. Figueiredo, Phys. Rev. E 60, 5367 (1999).

[17] S. Bekhechi et al. Phys. Rev. E, 64, 016134 (2001).

[18] M. Godoy and W. Figueiredo, Phys. Rev. E 65, 026111 (2002).

[19] Han Zhu, Jian-Yang Zhu, and Yang Zhou, Phys. Rev. E 66, 036106 (2002).

[20] Jian-Yang Zhu and Z. R. Yang, Phys. Rev. E 59, 1551(1999); 61, 210 (2000); 61, 6219 (2000).

[21] Han Zhu and Jian-Yang Zhu, Phys. Rev. E 66, 017102 (2002); Jian-Yang Zhu and Han Zhu, Chinese Phys. Lett. 19, 1669 (2002).

\section{Caption of figures}

Fig. 1 The phase diagrams of the three-dimensional Gaussian model on (a) regular lattice, (b) A-SWN, and (c) R-SWN. The regions Para, Ferro, and Hetero correspond to the paramagnetic, ferromagnetic, and heterophase phase, respectively, while the part of oblique lines belong to unknown region. 
(a) Regular-Type, $P_{A}=P_{R}=0$

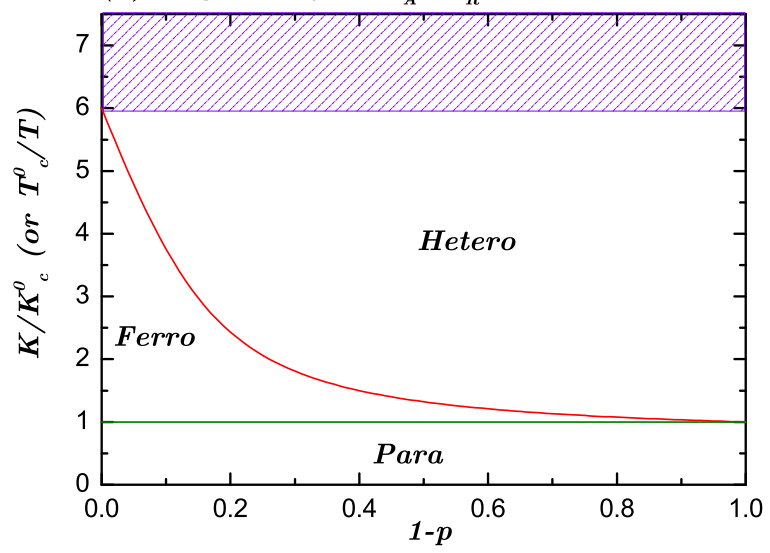

(b) Adding-Type $S W N, P_{A}=1 / N$

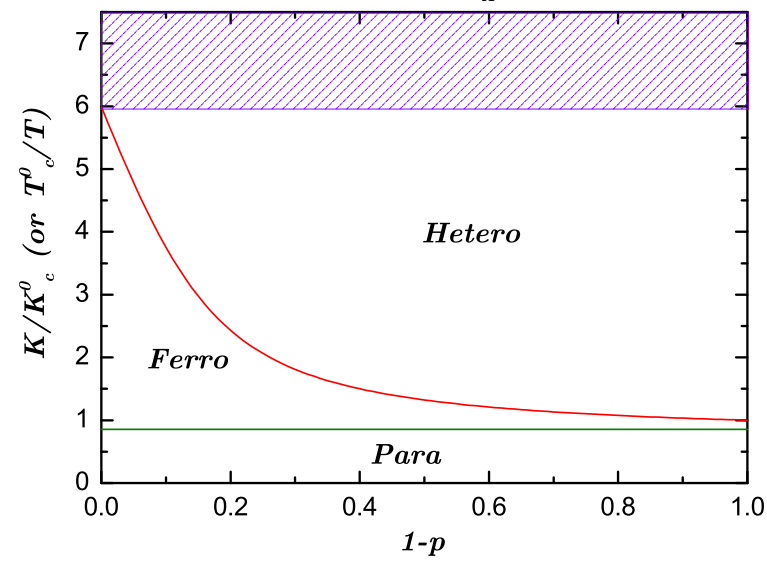

(c) Rewiring-Type $S W N, P_{R}=0.1$

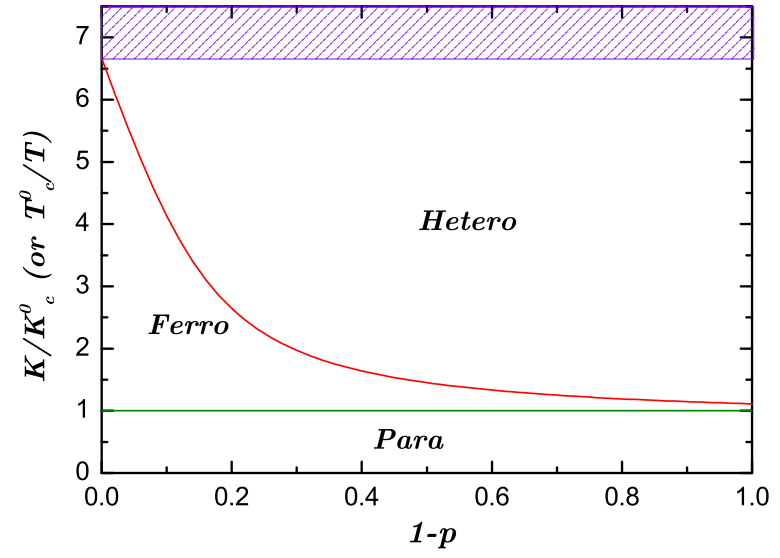

